\title{
Pharmacokinetic Concentration Elapsed Time
}

National Cancer Institute

\section{Source}

National Cancer Institute. Pharmacokinetic Concentration Elapsed Time. NCI Thesaurus.

Code C87954.

The interval between two pharmacokinetic concentration reference time points. 